\title{
Corrigendum: Photoinitiated charge separation in a hybrid titanium dioxide metalloporphyrin peptide material
}

H. Christopher Fry, Yuzi Liu, Nada M. Dimitrijevic \& Tijana Rajh

Nature Communications 5:4606 doi: 10.1038/ncomms5606 (2014); Published 18 Aug 2014; Updated 2 Feb 2015

The original version of this Article contained an error in the spelling of 'Photoinitiated' in the title of the paper. This has now been corrected in both the PDF and HTML versions of the Article. 